\title{
Tonal Patterns of Lisu Language
}

\author{
$\mathrm{Na} \mathrm{Li}{ }^{1}$ \\ ${ }^{1}$ The College of Arts and Sciences, Yunnan Normal University, Kunming, Yunnan Province, China
}

Keywords: Lisu language; tonal pattern; phonetic types.

\begin{abstract}
This paper adopts the method of acoustic experiment. Using computer and voice processing software, a comprehensive phonology analysis on tones of Lisu language is made to summarize and describe its phonetic features; a system of tonal pattern of Lisu language is established from the perspective of tonal features. Through collected experimental data, tonal charts which clearly represent adjustment patterns, duration of tones, tonal ranges and tone values of Lisu language are made by computer software. The tense and lax tones of Lisu language are analyzed from the angle of phonation modes. The author hopes to provide more detailed and accurate synchronic data on experimental study of tones of minority languages, and try to find out the tonal rules of Lisu language.
\end{abstract}

\section{Introduction}

Compared with Chinese dialects, linguistic scholars' experimental research on phonetic features of minority languages does not have a wide range. [1] But some deep and fruitful studies can also be found. In early years, many experts only focused on pitch when analyzing tones. What's more, when studying pitch, they mainly focused on adjustment patterns and tone values, and ignored the study of ranges and duration of tones. Later, the technique of experimental study on tonal pattern is gradually mature; experts begin to pay attention to duration of tones. But researches on refinement of tone ranges are not enough. [2] In recent years, a number of experts and scholars find another perspective of tonal study - phonation modes. Xiao-nong Zhu once said, "With the deepening of tonal study, we find that our previously thought is incorrect. Tones are only related with fundamental frequency, or the vibration speed of vocal cords. They also closely related with the phonation modes of vocal cords." [3] Physiologically speaking, there are six types of phonation mode, namely falsetto, fortis voice, voiceless whisper, harsh voice, breathy voice and creaky voice. The difference of phonation modes is reflected in the difference of spectral slope (H2-H1). In 1981, Bickly proposed that, the energy of fundamental frequency of the sound wave (first harmonic) is greater than second harmonic $(\mathrm{H} 1>\mathrm{H} 2)$. The energy difference between the first harmonic and the second harmonic of breathy voice is significantly greater than the energy difference between the first harmonic and the second harmonic of normal voice. [4] In 2001, Ladefoged proposed that the difference between the two is the main acoustic parameter that can distinguish different speech types in different languages. He also pointed out that in many languages, difference of lax vowels $>$ difference of normal vowels $>$ difference of creaky vowels. [5] [6]

\section{Lisu People, Orthography and Phonology of Lisu Language}

Lisu people and orthography of Lisu language. Lisu people mainly live in four counties, namely Lanping, Lushui, Fugong and Gongshan in Lisu Autonomous Prefecture of Nujiang River, Yunnan Province. They can also be found in other prefectures and provinces. Lisu language can be divided into three dialects of Nujiang River dialect, Luquan dialect and Ninglang dialect. The Nujiang River dialect is considered as the standard pronunciation. [7]

There are four kinds of orthography in Lisu language. The traditional Lisu orthography, created by Neng-ren Fu and Sai Ye Ba Duo; frame type alphabet, created by Hui-ren Wang; syllabic language, created by Ren-bo Wang; and the new Lisu orthography, issued by the government. [7] 
Phonology of Lisu language. There are 29 consonants in Lisu language, include [p, t, k, ph, th, kh, $\mathrm{b}, \mathrm{d}, \mathrm{g}, \mathrm{ts}, \mathrm{t} \int$, ts $\mathrm{h}, \mathrm{t} \int \mathrm{h}, \mathrm{dz}, \mathrm{d} 3 \mathrm{~s}, \mathrm{~m}, \mathrm{n}, \quad, \quad, \mathrm{l}, \mathrm{f}, \mathrm{s}, \int, \mathrm{x}, \mathrm{h}, \mathrm{v}, \mathrm{z}, \mathrm{z}, \mathrm{\gamma}, \mathrm{]}$. There are nineteen vowel phonemes in Lisu language. 7 vowels are simple vowels, include [i, e, $\varepsilon, a, o, u, u$ ]; 1 is apical vowel, namely [1 ]; 6 are nasal vowels, include $[\overline{\mathrm{e}}, \bar{\varepsilon} \quad, \overline{\mathrm{a}}, \tilde{\mathrm{o}}, \overline{\mathrm{u}}, \overline{\mathrm{u}} \quad$ ]; 5 are compound

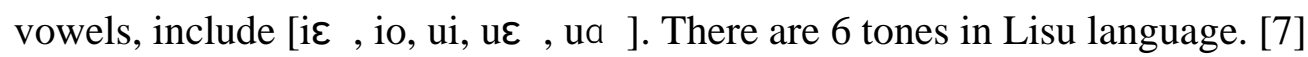

Tones of Lisu language. Brief Record of Lisu Language describes that Lisu language has 6 tones. There are 3 level tones, respectively the first 55 tone, the third 44 tone and the fourth 33 tone; one rising tone, namely the second 35 tone; 2 falling tones, include the fifth 42 tone and the sixth 31 tone. Among them, 44 and 33 tones, 42 and 31 tones can be transferred freely after voiceless constants; but they have strict distinctions after voiced constants. There is a certain relation between tones and the tightness of the vowels. Tense tones are 44 and 42 tones.

\section{Tonal Pattern of Lisu Language}

According to the analysis of experimental data, the chart on tonal patterns of Lisu language is obtained.

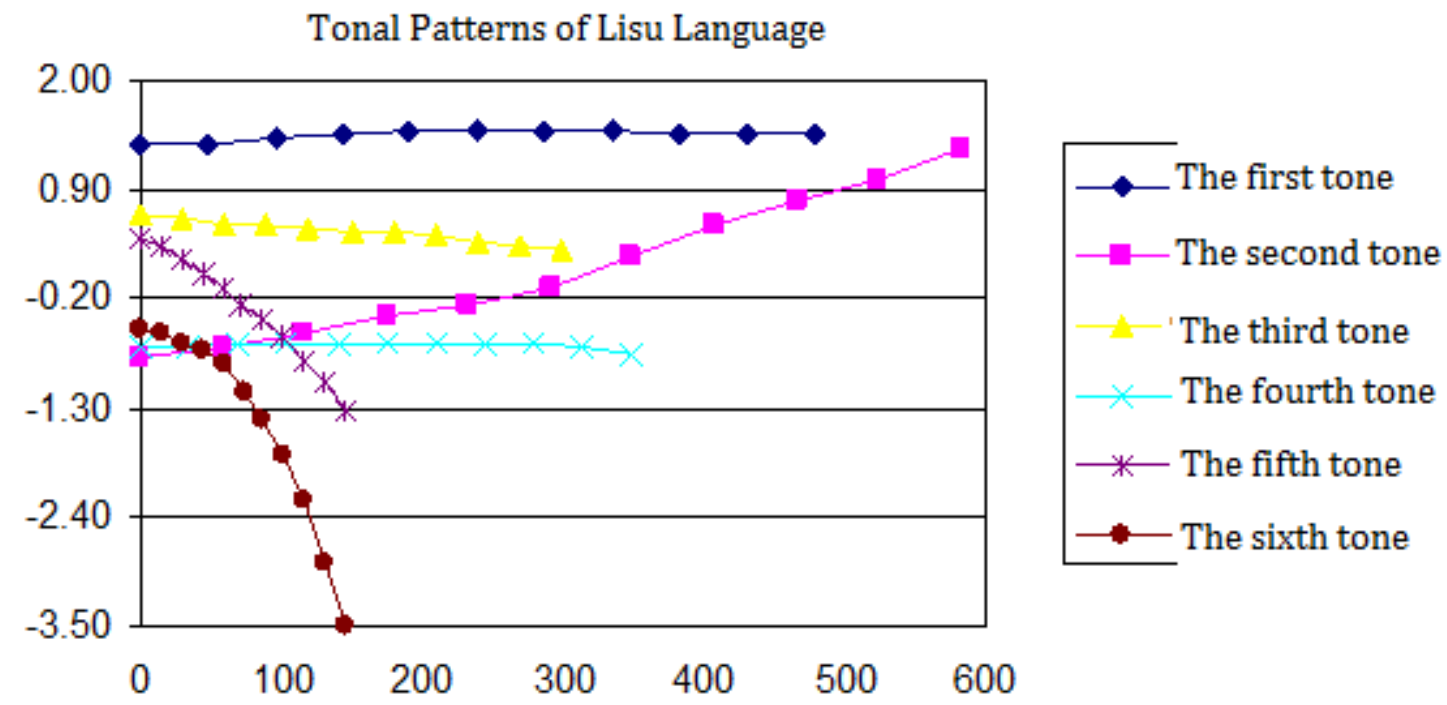

The traditional view holds that in Lisu language, the contrastive lax and tension vowels are the fourth tone (relative value 33, lax) and third tone (relative value 44, tension), as well as the sixth tone (relative value 31, lax) and the fifth tone (relative value 42, tension).

Adjustment patterns. One group of voice is composed of level tones; the other is composed of falling tones. This shows that, tension and lax vowels in Lisu language can be both level and falling tones; the contrastive tension and lax vowels have the same adjustment patterns. (If the lax vowel is level tone, its contrastive tension vowel is also level tone).

From the chart of tonal patterns of Lisu language, following characteristics on adjustment patterns of Lisu language can be found:

Tones in Lisu language are relatively simple. There is no falling-rising tone. Basic tones include level tone, rising tone and falling tone. The number of rising, level and falling tones are one, three and two.

In Lisu language, contrastive tension and lax vowels are level tones and falling tones.

There's a medium level tone, a high rising tone, a low falling tone and a sub-high falling tone in Lisu language. Contrastive tension vowels can be found in medium level tone. Lax vowels in low falling tones and tension vowels in sub-high falling tones are contrastive.

Tonal ranges. Tonal ranges are divided into tone categories and tone systems. Tone categories refer to variation ranges of specific pitch types from the highest tone to the lowest tone. Tone systems refer to variation range of the whole tone system from the highest tone to the lowest tone. The lowest pitch is the lower limit of tonal range; the highest is the upper limit. 
From the tonal patterns of Lisu language, it can be seen that the spans of tone categories of lax vowels in fourth and sixth tones are greater than the spans of tone categories of tension vowels in third and fifth tones.

The traditional view holds that, the contrastive lax and tension vowels are the fourth tone (relative value 33, lax) and third tone (relative value 44, tension), as well as the sixth tone (relative value 31 , lax) and the fifth tone (relative value 42, tension). Among the four tones, only the sixth tone reaches the lower limit of the tonal system. [8]

Tone values. From the Lisu language tonal patterns, it can be seen that there's no much difference between conclusions on adjustment patterns and tone values in this study and the traditional studies. Especially the tone values; the values of 6 tones are consistent with the traditional research. The values of the first, second, third, fourth, fifth and sixth tones are 55, 35, 44, 33, 42 and 31. This shows that the older generation of scholars and experts are very rigorous, and provide very accurate research data and valuable information for following research. It also proves the ability of our ears. Computer technology, though highly precise, cannot replace human ears so far. In addition, the processes of data selection, determination and analysis in a large number of phonetic experiments need the participation of human ears.

\section{Duration of tone.}

\begin{tabular}{|c|c|c|c|c|c|c|}
\hline $\begin{array}{c}\text { Type of } \\
\text { tones }\end{array}$ & First tone & $\begin{array}{c}\text { Second } \\
\text { tone }\end{array}$ & Third tone & $\begin{array}{c}\text { Fourth } \\
\text { tone }\end{array}$ & Fifth tone & Sixth tone \\
\hline $\begin{array}{c}\text { absolute } \\
\text { duration }\end{array}$ & 478 & 582 & 299 & 349 & 145 & 146 \\
\hline
\end{tabular}

The Relative Durations of Tones in Lisu Language

\begin{tabular}{|c|c|c|c|c|c|c|}
\hline $\begin{array}{c}\text { Type of } \\
\text { tones }\end{array}$ & First tone & $\begin{array}{c}\text { Second } \\
\text { tone }\end{array}$ & Third tone & $\begin{array}{c}\text { Fourth } \\
\text { tone }\end{array}$ & Fifth tone & Sixth tone \\
\hline $\begin{array}{c}\text { relative } \\
\text { duration }\end{array}$ & 1.43 & 1.78 & 0.89 & 1.05 & 0.42 & 0.43 \\
\hline
\end{tabular}

So, we can conclude the duration pattern of Lisu language as the following:

The second tone $>$ the first tone $>$ the fourth tone $>$ the third tone $>$ the sixth tone $>$ the fifth tone It can be seen that the relative durations of lax vowels are larger than that of the tension vowels.

Lax and tension tones. Pitch and duration. From the perspective of adjustment patterns, contrastive tension and lax vowels are in level tones and falling tones.

From the perspective of tonal ranges, in Lahu language, the spans of tone categories of lax vowels are greater than the spans of tone categories of contrastive tension vowels. This is the same with Lisu language. This shows that in the same language, the pronunciation of lax tones is sharper and shorter than contrastive tension tones.

From the perspective of duration, in the same language, the absolute and relative durations of lax tones are longer than contrastive tension tones. Thus, in the same language, durations of lax tones are longer than contrastive tension tones. It also shows that in the same language, the pronunciation of lax tones is sharper and shorter than contrastive tension tones.

Experimental study of spectral slope. Using Praat software, we extracted the first halves of each tension and lax vowels in the recorded pronunciation material of Lisu language, made FFT spectrum sections at about 50 milliseconds, and manually extracted the values of energy of the first and second harmonics (H1, H2). Then we imported data into Excel, and calculated the differences between H1 and H2. Obtained data are as follows: 


\begin{tabular}{|c|c|c|c|c|c|c|c|c|}
\hline \multicolumn{2}{|c|}{ Lax tones } & \multirow{2}{*}{$\frac{\text { h1 }}{25.60}$} & \multirow{2}{*}{$\frac{\text { h2 }}{17.00}$} & \multicolumn{2}{|c|}{ h1-h2 Tension tones } & \multirow{2}{*}{$\begin{array}{c}\text { h1 } \\
30.70\end{array}$} & \multirow{2}{*}{$\frac{\text { h2 }}{33.50}$} & \multirow{2}{*}{$\begin{array}{l}\mathbf{h} 1-\mathbf{h} 2 \\
-2.80 \\
\end{array}$} \\
\hline 4 & $(33)$ & & & 8.60 & (44) & & & \\
\hline 4 & $(33)$ & 38.10 & 23.10 & 15.00 & $(44)$ & 34.50 & 29.50 & 5.00 \\
\hline 4 & (33) & 37.20 & 27.60 & 9.60 & $3 \quad(44)$ & 26.70 & 24.60 & 2.10 \\
\hline 4 & (33) & 43.90 & 32.90 & 11.00 & 3: (44) & 23.50 & 21.40 & 2. 10 \\
\hline 4. & (33) & 43.10 & 25.40 & 17.70 & 3. (44) & 31.90 & 23.20 & 8. 70 \\
\hline 4 & (33) & 33.00 & 22.00 & 11.00 & $3^{\circ}(44)$ & 33.20 & 30.30 & 2. 90 \\
\hline 6 & $(31)$ & 25.70 & 13.00 & 12. 70 & 5. (42) & 19.20 & 17.90 & 1. 30 \\
\hline 6. & $(31)$ & 31.80 & 11.40 & 20.40 & $5 \quad(42)$ & 32.90 & 27.00 & 5.90 \\
\hline 6. & (31) & 30.50 & 21.60 & 8.90 & 5. (42) & 31.80 & 27.00 & 4. 80 \\
\hline $6 i$ & (31) & 27.70 & 11.00 & 16. 70 & $(42)$ & 42.20 & 29.20 & 13.00 \\
\hline 6. & (31) & 33.60 & 22. 40 & 11.20 & 5. (42) & 34.60 & 25.80 & 8.80 \\
\hline 6 & (31) & 35.60 & 26.10 & 9.50 & $5 \quad(42)$ & 19.10 & 11.60 & 7.50 \\
\hline Yean & value & 33.82 & 21.13 & 12. 69 & Mean value & 30.03 & 25.08 & 4. 94 \\
\hline
\end{tabular}

From the table, we can clearly see that the differences between $\mathrm{H} 1$ and $\mathrm{H} 2$ of lax tones in Lisu language are always positive. The mean value of differences between $\mathrm{H} 1$ and $\mathrm{H} 2$ is also positive. This shows that in lax vowels of Lisu language, the energy value of first harmonic is greater than the second harmonic.

Overwhelming majority of differences between $\mathrm{H} 1$ and $\mathrm{H} 2$ of tension tones in Lisu language is positive. There's only one exception. The mean value of differences between $\mathrm{H} 1$ and $\mathrm{H} 2$ is also positive. This shows that in tension vowels of Lisu language, the energy value of first harmonic is greater than the second harmonic, too.

We can see the comparison result of Lisu language in the spectral slope: lax tones are greater than tension tones. However, from the comparison of mean values of differences between H1 and H2, tension tones are much greater than lax tones.

It can be concluded that, Lisu vowels in tension tones belong to normal vowels, while vowels in lax tones belong to relaxed vowels.

This paper does the very first research on tonal patterns of Lisu language, a branch of Tibeto-Burman language, through traditional and experimental methods in history. [9] Adopting the method of acoustic experiment, and taking the perspective of tonal features, this paper tries to clearly represent the adjustment patterns, duration of tones, tonal ranges and tone values of Lisu language. Every language and dialect has its unique adjustment patterns, levels and numbers of tones, as well as its distribution relationship. These features form their special tonal patterns, and reflect the personalities of languages and dialects. Zong-ji Wu believes that, "tonal patterns can show the whole pictures of languages and dialects; they have similar meaning and values with 'landscapes' in geography” . [10] Hence, a large-scale research on tonal patterns of Sino Tibetan languages will enrich and promote the study of related types of tonal pattern, and provide a new point of view, as well as further evidence to the research on formation, development and changes of tones in each language, and the relationship between related languages. [11]

\section{References}

[1] F. Shi, R.R. Liao, The tonal pattern of Beijing dialect, in: Phonetics Study, Beijing Language and Culture University Press, Beijing, 1994

[2] F. Shi, R.R. Liao, Several issues on tone analysis, in: Phonetics Study, Beijing Language and Culture University Press, Beijing, 1994

[3] J.G. Peng, X.N. Zhu, Flasetto in Yueyang dialect, J. Contemporary Linguistics. 12 (2010) 24.

[4] F. Shi, R.R. Liao, Phonetics Study, Beijing Language and Culture University Press, Beijing, 1994 
[5] X.N. Zhu, X.W. Zhou, Cracking: tension vowels in Hani Language, J. Minority Languages of China. 4 (2008).

[6] Q.X. Dai, Study on Minority Languages in Twentieth Century, Suhai Books Press, Taiyuan, 1998

[7] L. Xu, Y.T. Mu, X.Z. Gai, Records of Lisu Language, The Ethic Publishing House, Beijing, 1986

[8] Compilation Committee of Yunnan, Minority Languages Committee of Yunnan Province, Yunnan Province Chorography, Volume Fifty-nine: Minority Language Records, Yunnan People's Publishing House, Kunming, 1997

[9] Q.X. Dai, Tibetan Burman Language Research, The Nationalities Publishing House of Yunnan, Kunming, 1990

[10] F. Shi, Experimental Phonology Research. Peking University Press, Beijing, 2009.

[11] School of Chinese Ethnic Minority Languages, Minzu University of China, Chinese Minority Languages, Sichuan Nationalities Press, Chengdu, 1987 